\title{
Design, Implementation, and Evaluation of an Information System to Enhance Road Safety Level for Pedestrians in China
}

\author{
Hanjing Huang, ${ }^{1,2}$ Luosha Liu, ${ }^{3}$ Zhiyong Fu, ${ }^{4}$ Yichi Zhang, ${ }^{3}$ Jun Zhang, ${ }^{4}$ Yuehlin Tsai, ${ }^{2}$ \\ Li Guo, ${ }^{4}$ and Pei-Luen Patrick Rau $\mathbb{D D}^{2}$
}

${ }^{1}$ School of Economics and Management, Fuzhou University, Fuzhou, China

${ }^{2}$ Department of Industrial Engineering, Tsinghua University, Beijing, China

${ }^{3}$ Siemens Ltd., Beijing, China

${ }^{4}$ Department of Information Art \& Design, Tsinghua University, Beijing, China

Correspondence should be addressed to Pei-Luen Patrick Rau; rpl@mail.tsinghua.edu.cn

Received 31 March 2021; Revised 22 September 2021; Accepted 20 October 2021; Published 17 November 2021

Academic Editor: Floriano Scioscia

Copyright (c) 2021 Hanjing Huang et al. This is an open access article distributed under the Creative Commons Attribution License, which permits unrestricted use, distribution, and reproduction in any medium, provided the original work is properly cited.

\begin{abstract}
Pedestrians' unsafe behavior is one of the most critical factors causing traffic incidents in China. The primary objective of this study is to explore the cause of pedestrians' unsafe behavior and provide possible solutions. We interviewed pedestrians and experts to investigate pedestrians' unsafe behaviors. Results from interviews indicated that pedestrians were likely to exhibit unsafe behavior at intersections owing to use of smartphones, reluctance to obey the rules, and unawareness of risk. According to the experts, attracting the attention of pedestrians and guiding them to exhibit safe behaviors can improve their safety. Based on these results, we designed "LookMe," which is a multimedia information system placed at the intersections, to guide pedestrians across the road and improve their experience of waiting in traffic. The results of user tests indicated that pedestrians had relatively high acceptance of LookMe. Moreover, participants wanted to see diverse multimedia information on the screen of LookMe such as news, videos, maps, and traffic information. Findings from this study can be useful in understanding why Chinese pedestrians exhibit unsafe behaviors and proposing effective solutions to enhance their safety.
\end{abstract}

\section{Introduction}

Pedestrian safety has been considered as an important problem worldwide. Over 27,300 pedestrians were killed in traffic crashes, which accounted for $22 \%$ of total fatalities globally in 2010 [1] (see Figure 1). The problems of pedestrian fatalities are even more serious in developing countries such as China. Road traffic death in China accounts for $23 \%$ of the global total $[2,3]$. Pedestrians are at a higher risk than drivers and passengers in traffic accidents owing to their fragility and slow movements [4]. Drivers need to keep a certain route, whereas pedestrians are more free to move around [5]. Pedestrians are less restricted by regulations, and their behaviors are often unpredictable. Factors leading to pedestrian crashes and deaths include human factors, infrastructure, and environment [6].
Pedestrians' improper crossing the road, distraction, and failure to obey traffic rules significantly contribute to pedestrian crashes and death. These unsafe behaviors account for $28 \%, 15 \%$, and $3 \%$ of pedestrian death [7].

In China, pedestrian crashes are more likely to occur at intersections. Previous studies have reported that pedestrians' red-light running behavior is a main cause of pedestrian traffic accidents at the intersections [6]. The pedestrians' red-light running behavior is widespread in China $[8,9]$. The red-light running behavior can be affected by many factors such as the user characteristics (e.g., demographic variable), the physical environment (e.g., design of road), and the social factors (e.g., the number of pedestrians) [10]. For example, elderly pedestrians are less likely to run the red light because they are more cautious. When pedestrians have to wait longer, they are more likely 


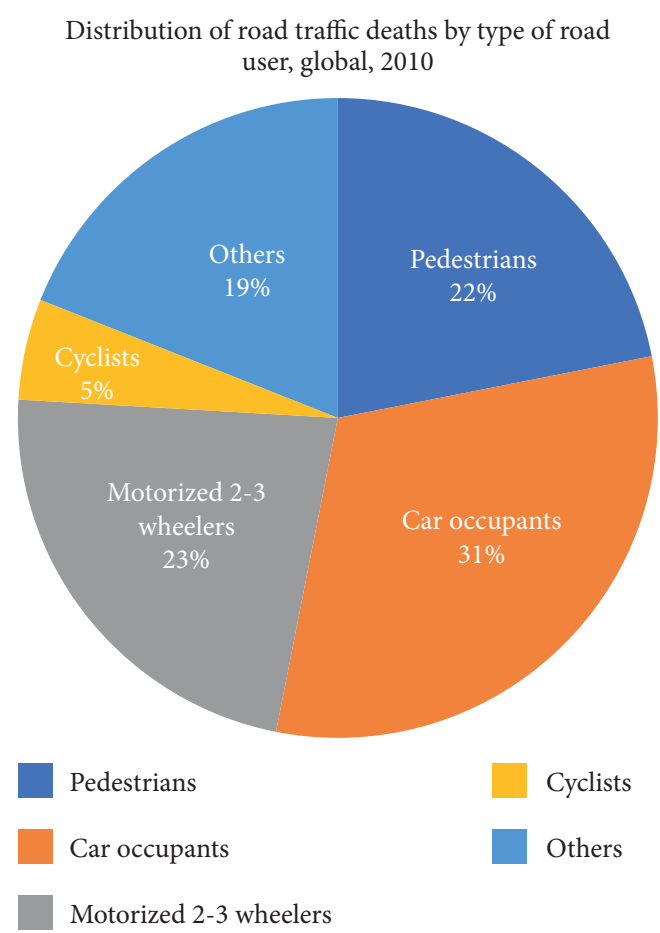

FIGURE 1: Distribution of road traffic deaths by type of road user in 2010.

to run the red light [11]. Pedestrians in a hurry are more likely to run the red light. In addition, pedestrians tend to cross on red more often along with others. Researchers also suggested that the intersections in China should be improved from a more user-oriented perspective, as pedestrians were not satisfied with current intersections [10].

Pedestrians' distraction is another factor threatening pedestrians' safety. Pedestrians have always engaged in multiple tasks like listening to music, text messaging, and using a handheld phone that draw their attention while walking, even while crossing the road. Nearly one-third of all pedestrians performed a distracting activity while crossing [12]. In China, mobile phone use while crossing the road is prevalent (i.e., 53\%) [13]. Many accidents that resulted from pedestrians' distraction have been reported $[7,14,15]$. Pedestrians using mobile phones while crossing were more likely to be hit by oncoming vehicles at intersections [16]. Distracted pedestrians adopt less cautionary behavior (e.g., looking left and right or waiting for a walk signal) before crossing the road compared to other pedestrians. Mobile phone use is associated with fewer cautionary behaviors and slower crossing times [12]. The experiments in a virtual reality environment also found that pedestrians were less able to recognize safe opportunities to cross the road when they were conversing on the mobile phones [17]. Pedestrians who are texting messages were 3.9 times more likely to exhibit unsafe crossing behaviors compared to undistracted pedestrians [18]. These researches have provided substantial evidence for the harmfulness of pedestrian distraction. However, distracted walking has received fewer policies and effective interventions than distracted driving did. There is a need for more innovations and technologies to combat distracted walking $[12,18]$.

Previous researchers have highlighted the importance of using technologies to reduce pedestrians' running red lights and distracted walking. Some researchers proposed intelligent systems for pedestrians, such as WalkingSafe [19], UltraSee [20], Surround-See [21], and Smartroad [22]. Most of these systems mainly rely on the detection of surrounding environments or alerting the pedestrians of the hazards on the mobile phone. For example, UltraSee was proposed to augment mobile phones with a small ultrasonic sensor to detect the abrupt change of the ground for pedestrians [20]. These systems are mainly applications installed in mobile phones. The pedestrian's attention is still on the mobile phone. There is a lack of user tests on the effectiveness of these systems to reduce pedestrians' unsafe behaviors or to enhance pedestrians' safety. The Safe System approach emphasized using an integrated approach, which considered multiple factors involved in pedestrian safety, such as road design, vehicle speed, and enforcement of traffic laws and regulations $[1,23]$. The Safe System approach provided a basis for low- and middle-income countries to improve infrastructures for pedestrians and pay adequate attention to pedestrians' needs.

This study aimed to find a new solution that could encourage pedestrians to exhibit safe behaviors through optimizing the information presentations at intersections and diverting the attention of pedestrians from their mobile phones based on the user-centered design.

\section{Methodology}

This study followed the steps of the user-centered design to explore the possibilities of using information systems to improve pedestrians' safety behaviors. The specific steps of the study are presented in Figure 2: (1) The interviews included the interview of pedestrians and the interview of experts. The interview of pedestrians was conducted to gain information about pedestrians' unsafe behaviors and the causes of the unsafe behaviors. The interview of experts was conducted to analyze the pedestrians' behaviors, the possible solutions, and future directions. (2) Based on the results of interviews, we proposed a multimedia information system placed at the intersections to guide pedestrians to have more safe behaviors. (3) The user tests followed in order to investigate the feasibility of the information system.

\section{Interviews}

This study used the user-centered design to propose more effective systems to improve their safety in China. The interviews of pedestrians and experts were conducted to investigate pedestrians' behaviors, need, causes of unsafe behaviors, and possible directions of solutions. The sample sizes of the interviews were determined by the BJHP rule that stated that a sample of eight participants was appropriate for the exploratory researches focusing on identifying the 


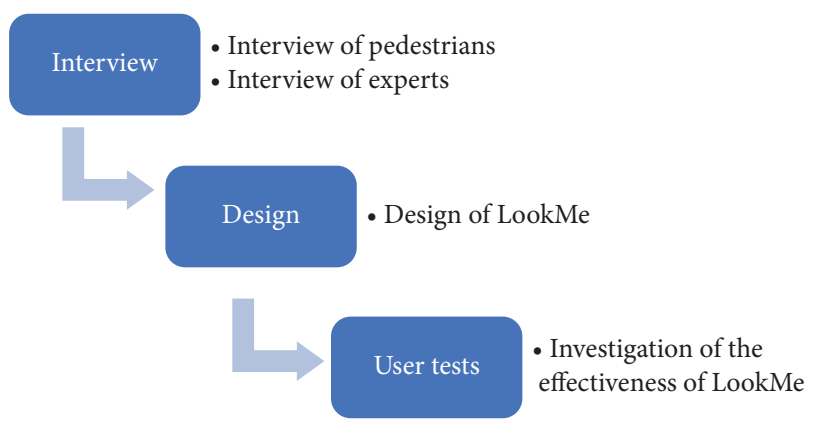

Figure 2: Overview of the methods.

underlying ideas about the topic [24]. In this study, eight pedestrians and nine experts participated in the interviews.

3.1. Interviews of Pedestrians. The aim of the interview of pedestrians was to investigate the current status of pedestrians' unsafe behaviors in crossing the road and walking in China. In addition, design of the traffic light and the sidewalk, effects of bicycles and electronic bicycles, and attitudes of traffic laws were investigated. The interview questions were based on previous related research and reviewed by an expert panel. A pilot study was conducted to refine the questions before the interviews were held. The following research questions were examined:

(1) What are pedestrians' unsafe behaviors?

(2) Do the designs of software and hardware facilities meet pedestrians' needs?

(3) What are the effects of the bicycle and the electronic bicycle on pedestrians' safety?

The recruitment flyers were posted in social media and distributed to pedestrians on the road. Pedestrians interested in this topic could sign up for interviews. In all, eight pedestrians participated in the interview. Participants included students, office workers, parents, older people, and parents of children. All interviews were conducted in the usability lab and lasted for approximately one hour. Interviews with different participants were conducted separately. The participant came to the usability lab individually at the appointed time. Two staff members were involved in the interview sessions: one was responsible for hosting each interview and the other handled audio recording. Each interview was audio-taped and transcribed verbatim. The interview data were then analyzed using a structured process: open coding, axial coding, and selective coding. The details of results are listed in Table 1.

Participants' answers were clustered into three subsections: unsafe behaviors, design of software and hardware facilities, and effects of bicycles and electric bicycles.

For unsafe behaviors, most pedestrians (6/8) had experience of running the red light. They would judge whether they could pass through an intersection based on the traffic flow and the traffic light. Pedestrians often followed the crowd to cross the road. Pedestrians who did not have experience of running the red light emphasized more on safety $(2 / 8)$. In addition, it was common for pedestrians to use mobile phones while waiting for the green light and walking. Most pedestrians (5/8) watched their mobile phones while waiting for the green light. Although pedestrians realized that watching mobile phones would affect their observations of changes in traffic light and pose a safety risk, most of them were interested in watching their mobile phones. Meanwhile, most pedestrians had the experience of watching the mobile phones while walking on the sidewalk (7/8). Most of them did not think this behavior was dangerous. For attitudes toward traffic laws, all pedestrians $(8 / 8)$ believed that abiding by traffic rules was essential. However, the majority was just aware of the following traffic rules: "red light stop, green light go, walk zebra crossings." Half of pedestrians (4/8) did not know that they would be penalized for violating traffic rules.

For design of software and hardware facilities, most pedestrians claimed that the current design could not meet their needs. For the design of traffic light, most pedestrians (7/8) expected that the display of the remaining time could be added to traffic light. Some pedestrians (3/8) expected the traffic light to include voice prompts, but other pedestrians (5/8) assumed that the voice prompts would generate noise. Half of pedestrians thought that the current sidewalk was not wide enough $(4 / 8)$. In addition, most pedestrians were not aware of the right area for waiting for the green light (6/8). Most pedestrians (7/ 8 ) chose to stand behind the crowd, and one pedestrian (1/8) chose the middle position. To ensure safety and avoid conflicts with others, no pedestrian chose the front position.

For the effects of bicycles and electric bicycles, pedestrians claimed that bicycles and electric bicycles would affect their walking and safety. In walking, most of the pedestrians were affected by bicycles and electric bikes parked on the sidewalk (7/8). The parking of bicycles and electric bicycles also made the sidewalk narrow and untidy. In addition, most of the pedestrians thought that pedestrians might have conflicts with bicycles and electronic bikes on the sidewalk (7/8). For example, electronic bikes might run to the front and back suddenly.

In summary, results of pedestrians' interviews indicated that most pedestrians had experience of running red lights. They were interested in watching their smartphones while waiting and walking. The design of traffic lights and sidewalks needs to be optimized. Most pedestrians were unsure about the exact area for waiting. Random parking of bicycles and electronic bicycles would make the sidewalk narrow and untidy. There may exist conflicts between bicycles and pedestrians on the road. Pedestrians knew that they needed to obey the traffic rules, but they were not very clear about the specific traffic rules and punishment mechanisms.

3.2. Interviews of Experts. The aim of the interview of experts was to investigate the importance of pedestrian issues, the causes of conflicts, and the possible direction of solutions. The interview questions were also based on previous related research and reviewed by an expert panel. A pilot study was conducted before the interviews were held. The following research questions were investigated: 
TABLE 1: Clusters of pedestrians' answers.

\begin{tabular}{|c|c|}
\hline Subsections & Examples \\
\hline \multicolumn{2}{|l|}{ Unsafe behaviors } \\
\hline Behavior of running red lights & $\begin{array}{c}\text { "Sometimes, I don't pay attention to the traffic light when I think about other things, and I } \\
\text { just follow the crowd." (Pedestrian \#2) } \\
\text { "Because everyone goes over." (Pedestrian \#4) } \\
\text { "When there's no car in sight, I would run the red light." (Pedestrian \#5) }\end{array}$ \\
\hline Use of mobile phones at the intersections & $\begin{array}{l}\text { "I would watch the phone and sometimes, I failed to notice when the green light was already } \\
\text { on." (Pedestrian \#4) } \\
\text { "I would watch mobile phone and sometimes it would affect my observation of traffic lights." } \\
\text { (Pedestrian \#8) }\end{array}$ \\
\hline Use of mobile phones while walking & $\begin{array}{l}\text { "I fell over." (Pedestrian \#2) } \\
\text { "I don't think it's dangerous. There's no danger." (Pedestrian \#5) } \\
\text { "Sometimes, but no dangerous situation has occurred." (Pedestrian \#6) }\end{array}$ \\
\hline $\begin{array}{l}\text { Unaware of the right area for waiting for } \\
\text { the green light }\end{array}$ & $\begin{array}{l}\text { "I am unsure about the area to wait." (Pedestrian \#1) } \\
\text { "I don't know where to stand, and therefore, I usually stand behind." (Pedestrian \#6) } \\
\text { "Being in the middle because the middle is safer. I won't be hit by a turning vehicle, and I can } \\
\text { pass through faster in the middle." (Pedestrian \#7) }\end{array}$ \\
\hline Attitudes toward traffic laws & $\begin{array}{l}\text { "Pedestrians won't be punished for running the red light." (Pedestrian \#3) } \\
\text { "If there is punishment, situation will be better." (Pedestrian \#8) }\end{array}$ \\
\hline \multicolumn{2}{|l|}{ Design of software and hardware facilities } \\
\hline${ }_{0}$ & $\begin{array}{l}\text { "Displays the remaining time." (Pedestrian \#5) } \\
\text { "Audible prompts are required because sometimes it is hard to see traffic lights." (Pedestrian } \\
\# 4)\end{array}$ \\
\hline Uptimization of traftic light design & $\begin{array}{l}\text { "I don't know how long it takes." (Pedestrian \#7) } \\
\text { "Sometimes I can't see the traffic lights clearly, because the bus will block them." (Pedestrian } \\
\# 3 \text { ) }\end{array}$ \\
\hline Optimization of sidewalk & $\begin{array}{c}\text { "It doesn’t seem wide enough at present. There are more and more cyclists. I Feel very } \\
\text { crowded." (Pedestrian \#8) } \\
\text { "Some places are enough, some places are not enough." (Pedestrian \#4) }\end{array}$ \\
\hline
\end{tabular}

Effects of bicycles and electric bicycles

"Some people park their bicycles on the sidewalk, which may have some problems in safety." (Pedestrian \#6)

"Bicycle parking makes way for a narrower road." (Pedestrian \#7)

Conflicts with bicycles and electric bicycles

"Random bicycle parking affects the overall appearance and mood." (Pedestrian \#4)

"There are a lot of electric bikes and bicycles. There are many cyclists. It's really crowded." (Pedestrian \#8)

"Sometimes, suddenly, there is a bicycle or electric bike in front and behind." (Pedestrian \#7) "Bicycles can cause some avoidance or safety problems." (Pedestrian \#6)

(1) What is the importance of pedestrian issues?

(2) What are the causes of conflicts?

(3) What are the possible solutions and future directions?

The invitation of experts was based on the social representation approach, which focused on experts' research interests and accomplishments. The expert interviews are appropriate for explorative and product generation purposes [25]. Experts who were pioneers provided invaluable insights to help us better understand possible solutions and future directions of enhancing safety of pedestrians. First, we searched the research institutes conducting researches and projects about transportation and pedestrians to select the potential participants. We searched on the websites to acquire contact information of potential participants. We sent e-mail invitations of participation to all potential candidates, which included the overview of this study. Upon approval of the participant, we negotiated with the participant about the time and the place of the interview. In all, nine experts participated in the interviews. Research backgrounds of experts included intelligent transportation, car engineering, signals control, civil engineering, transportation plan, automobile crash, and human factors. Results of pedestrians' interviews highlighted the importance of reducing pedestrians' unsafe behaviors, optimization of the software and hardware designs, and reducing the effects of bicycles and electronic bicycles. The research backgrounds of experts were highly related to the improvement directions suggested by the pedestrians' interviews. The interview lasted about one hour. Interviews with different experts were conducted separately. During the interview, one staff member was responsible for hosting each interview and the other staff member handled audio recording in the interview. The 
interviews were recorded and the responses were transcribed verbatim. Analysis of interview data followed structured process: open coding, axial coding, and selective coding. The details of the results are listed in Table 2.

Experts' answers were clustered into three subsections: importance of pedestrian issues, causes of conflicts, and possible solutions to pedestrian issues.

For the importance of pedestrian issues, most experts (7/9) believed that pedestrians required special attention in transportations. Some experts (5/9) pointed out that pedestrians brought out some conflicts in transportation. Pedestrians often did not obey the traffic rules. Other experts advocated more measures to enforce the safety of vulnerable pedestrians (3/9). All the people who left the vehicles were pedestrians; therefore, the safety of pedestrians was the most important.

For the causes of conflicts, experts mainly pointed out two reasons. One reason was that the current design did not meet people's needs. For example, the sidewalk was too narrow that pedestrians needed to walk to the motorway. The other reason was that pedestrians' behaviors were unpredictable. Pedestrians were highly mobile individuals and often did not obey the traffic rules. For example, pedestrians often walked through the streets randomly. The punishment mechanisms of pedestrians' unsafe behaviors were also not complete.

For the possible solutions to pedestrian issues, experts claimed that it would be beneficial if more guidance was provided to encourage pedestrians to pay more attention to the traffic light and obey the traffic rules. The most frequently mentioned methods included "collecting information of pedestrians and vehicles and using immediate notifications" (5/9).

In summary, the interview revealed important phenomena and potential ways to improve pedestrians' safety. First, the interviews of pedestrians provided some important findings: most pedestrians had experiences of running the red light; most pedestrians liked to watch mobile phones when waiting and walking; most pedestrians were unaware of the right area for waiting and the traffic rules to be followed. The device at the intersections should offer more information. There are electronic bicycles and bicycles on the sidewalk. The sidewalk should be larger. Second, the interviews of experts provided some valuable results: most experts believed that pedestrians required special attention in transportations; the unsafe behaviors of pedestrians would lead to conflicts between vehicles and pedestrians; more products and measures were needed to guide pedestrians to conduct safe behaviors. Based on the results, it is possible to use information systems to guide pedestrians to conduct safe behaviors.

\section{Design of LookMe: Pedestrian-Centered Information System}

The results of interviews revealed that pedestrians exhibited unsafe behaviors such as use of smartphones, reluctance to obey the rules, and unawareness of risk. Experts pointed out that it would be beneficial to use more products and measures to guide pedestrians for enforcing safe behaviors. Using an information system to attract and guide them to exhibit safe behaviors can be a possible direction toward enhancing pedestrian safety. Therefore, we proposed an information system to encourage pedestrians to obey traffic rules and to increase their safety. The information system is called "LookMe" (see Figure 3). LookMe is designed to be placed at the intersections, as pedestrian crashes are more likely to occur at intersections in China. When LookMe is placed at the intersection, it can cover more pedestrians. In addition, pedestrians have time to watch LookMe when waiting for the green light at the intersection. LookMe includes different types of multimedia information to attract pedestrians' attention. When the traffic light is red, LookMe will represent multimedia information to reduce their boredom while waiting. When the traffic light turns green, the information system turns to tell the pedestrians to cross the road. When pedestrians watch LookMe, they also pay attention to the change of traffic light information, which can reduce distraction caused by mobile phones. The information displayed on LookMe should appeal to pedestrians and meet pedestrians' needs. Meanwhile, a pedestrian may follow others to watch LookMe affected by the herd mentality, as there are many people at the intersections. In addition, pedestrians can scan the QR code on the device to obtain bonuses when they are detected as waiting for the green light rather than running the red light. The program can also be used to collect other pedestrians' safe behaviors. For example, the program can record the time and frequency of pedestrians looking at mobile phones at sidewalks and intersections. With less use of mobile phone, they gain more tokens in the program. Therefore, LookMe may have positive effects on pedestrians' safe behaviors. The key design functions of LookMe are listed in the following.

4.1. Data Acquisition. The sensors are used to acquire information about the pedestrian volume, vehicle volume, traffic situations, and traffic accidents.

4.2. Traffic Information and Alerts. Pedestrians can always see the traffic signals on the screen. When the traffic light turns green, the full screen would tell the pedestrians to cross the road.

4.3. Multimedia Information. When the traffic light is red, pedestrians can have access to information on the screen such as news, weather information, maps, videos, and advertisements.

4.4. Additional Functions. Pedestrians can scan the QR code on the device to obtain bonuses when they are detected as waiting for the green light rather than running the red light. The program can also be used to collect time and frequency of pedestrians looking at mobile phones at sidewalks and intersections. Pedestrians' tokens were positively correlated with their safe behaviors.

4.5. Help. Pedestrians can use the system to navigate or call the police. 
TABLE 2: Clusters of experts' answers.

\begin{tabular}{|c|c|}
\hline Subsections & Examples \\
\hline \multicolumn{2}{|l|}{ Importance of pedestrian issues } \\
\hline \multirow[b]{2}{*}{ Large number of pedestrians } & $\begin{array}{c}\text { "All the people who leave the vehicles are pedestrians, so I think pedestrian facilities are the most } \\
\text { important." (Expert \#5) }\end{array}$ \\
\hline & $\begin{array}{l}\text { "Pedestrians are vulnerable groups. When they are involved in accidents, they are the most vulnerable. } \\
\text { More measures should be taken to protect pedestrians." (Expert \#7) }\end{array}$ \\
\hline & $\begin{array}{c}\text { "Traffic accidents are relatively complicated and confusing because there are many people who do not } \\
\text { follow the rules." (Expert \#2) }\end{array}$ \\
\hline Pedestrians' unsafe behaviors & $\begin{array}{c}\text { "When pedestrians cross the road, looking down at their mobile phones and not observing the road } \\
\text { conditions often lead to accidents or conflicts between motor vehicles, non-motor vehicles and } \\
\text { pedestrians." (Expert \#6) }\end{array}$ \\
\hline \multicolumn{2}{|l|}{ Causes of conflicts } \\
\hline Current design & $\begin{array}{l}\text { "The problem of pedestrians also starts from the aspect of design. For example, why do pedestrians walk } \\
\text { to the motorway? I think it is because there are too many pedestrians, and the sidewalk cannot meet the } \\
\text { needs of pedestrians." (Expert \#5) }\end{array}$ \\
\hline $\begin{array}{l}\text { Pedestrians' unpredictable } \\
\text { behaviors }\end{array}$ & $\begin{array}{l}\text { "Pedestrians are highly mobile individuals, and the unpredictability of pedestrian behaviors would lead } \\
\text { to conflicts." (Expert \#1) } \\
\text { "The major reason is that pedestrians don't obey the traffic rules. Pedestrians often walk through the } \\
\text { streets randomly." (Expert \#2) } \\
\text { "The punishment mechanism of pedestrians is not complete." (Expert \#8) }\end{array}$ \\
\hline \multirow{2}{*}{\multicolumn{2}{|c|}{$\begin{array}{l}\text { Possible solutions to pedestrian } \\
\text { issues }\end{array}$}} \\
\hline & \\
\hline Optimization of design & $\begin{array}{c}\text { "Maybe a clear indication may improve the current situation. We can add the signs waiting for the green } \\
\text { light zone." (Expert \#1) }\end{array}$ \\
\hline $\begin{array}{l}\text { Guide pedestrians' safe } \\
\text { behaviors }\end{array}$ & $\begin{array}{l}\text { "They did not proceed in a safe manner for two reasons: the first was that they were unaware about safe } \\
\text { behaviors, and the second was that they were aware but did not follow safe behaviors. More products } \\
\text { were required to help people understand the importance of safe behaviors." (Expert \#2) } \\
\text { "Use effective teaching methods to encourage pedestrians obey the traffic rules rather than simply } \\
\text { enforce punishment." (Expert \#7) }\end{array}$ \\
\hline
\end{tabular}

\section{User Tests}

Eighteen participants (11 males, 7 females) took part in the user tests. For usability tests, the " $10 \pm 2$ Rule" [26] suggested a sample size of eight to twelve participants in usability tests. In this study, eighteen passers-by at the intersection were recruited as participants. Their ages ranged from 18 to 50 years. The age distribution was as follows: one below 18 years, fourteen between 18 and 25 years, one between 26 and 30 years, one between 31 and 40 years, and one between 41 and 50 years. All users were provided an incentive of 3 USD for their participation. Participants' most commonly used modes of travel were walking $(72.2 \%)$, taking the subway $(72.2 \%)$, taking a taxi $(61.1 \%)$, riding a bicycle $(61.1 \%)$, and riding an electric bicycle (55.6\%).

The prototype was printed as the roll screen placed at the intersections. The most important function of LookMe was to attract pedestrians to look at the information on it. Therefore, the aim of the user tests was to test whether this information system could lower people's willingness to run the red light and ease their stress of waiting in traffic. Moreover, we focused on the type of information pedestrians needed or were interested in. There were two prototypes. This user test followed within-subject design; namely, each participant needed to experience these two prototypes. The control prototype with only the traffic light information was placed at position $B$, whereas the experimental prototype with the multimedia information and traffic light information (LookMe prototype) was placed at position $\mathrm{C}$. The participants were randomly assigned to one of the two sequences to experience. Half of the participants crossed the road following the sequences, from $A$ to $B$, from $\mathrm{B}$ to $\mathrm{C}$, and from $\mathrm{C}$ to $\mathrm{D}$. They experienced the control prototype at first and the experimental prototype later. The other half of the participants crossed the road following the reverse sequences, from $D$ to $C$, from $C$ to $B$, and from $B$ to A. They experienced the experimental prototype at first and the control prototype later (see Figure 4). The sequences of LookMe prototype and control prototype were counterbalanced. At position $\mathrm{B}$ and position $\mathrm{C}$, they completed the questionnaires. The questionnaire was designed to collect feedback of user experience. The questionnaire (shown in Table 3) was adapted from previous research on media [27-29]. The questions have been adapted to fit the aspect of our scenarios. A 5-point Likert rating scale was adopted for answering the closed questions of the questionnaire.

We used paired $t$-tests to examine the effect of an information system on participants' experience and moods. Table 4 shows the comparison results. The results of the paired $t$-tests revealed that participants had significantly lower levels of boredom, perceived monotony of environment, and impatience with the multimedia information screen (all $p<$ $0.05)$, suggesting that the multimedia information screen 


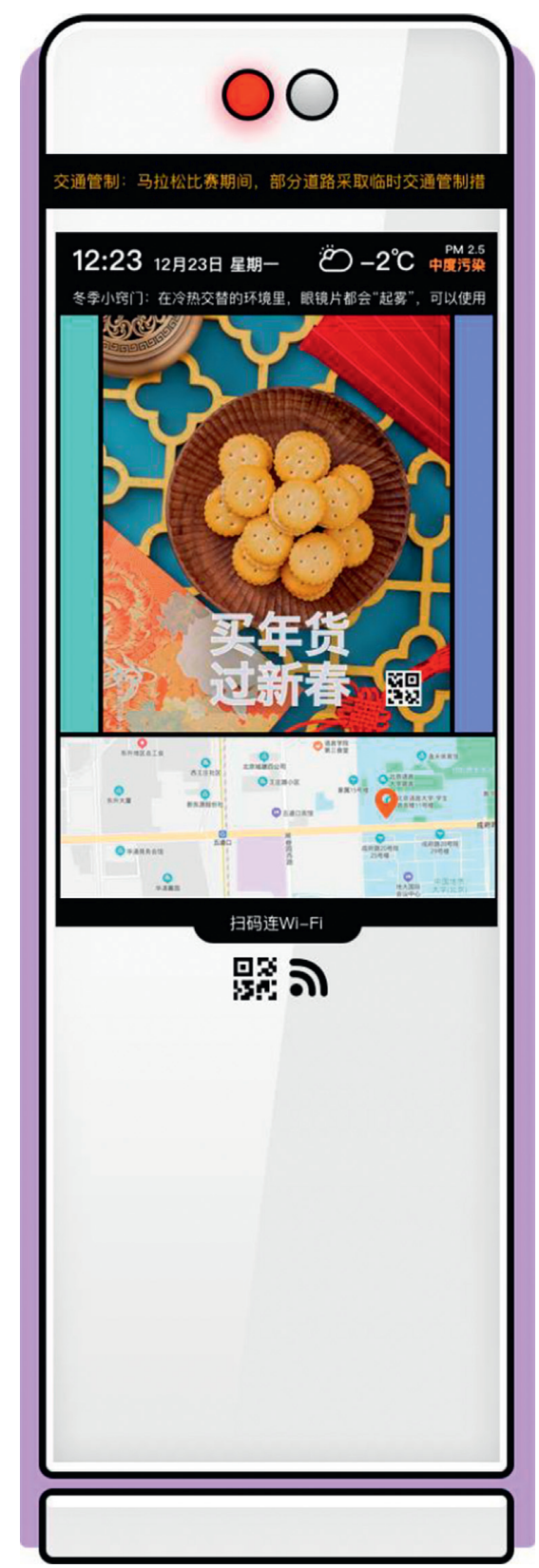

Figure 3: LookMe design.

could increase their experience of waiting for the green light. Meanwhile, participants rated an average score of 3.83 $(\mathrm{SD}=.71)$ on the 5 -point scale of willingness to look at the screen displaying multimedia information, indicating that participants were likely to look at the screen with multimedia information while waiting for the green light.

Furthermore, we analyzed participants' need for different types of information. The results showed that the most needed multimedia information is news, followed by weather and traffic information (see Figure 5). However, only $33.3 \%$ of participants thought that the advertisements needed to appear on the information screen.

Meanwhile, we used one-way ANOVA tests to analyze participants' evaluations of different types of information in meaningfulness, attractiveness, and willingness to use. The

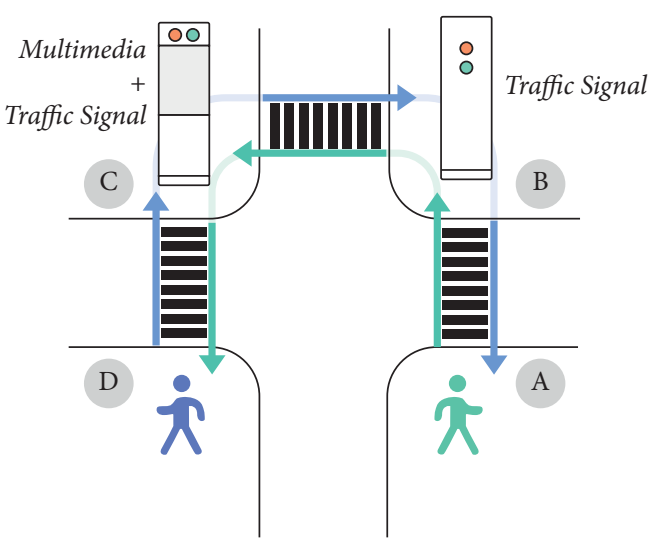

FIgURe 4: Environment of user tests.

results showed that the types of information had significant effects on participants' evaluations in meaningfulness, attractiveness, and willingness to use (all $p<0.05$ ). Furthermore, the results of post hoc analyses demonstrated that participants had significantly lower ratings of advertisements in meaningfulness, attractiveness, and willingness to use and preferred the screen of LookMe to not involve advertisements. For other types of information, participants did not show significant differences in their evaluations. Figure 6 showed participants' ratings of different types of information. The results suggested that participants had relatively high evaluations of the information, as all types of information had an average of the score above 3 points on the 5-point scale in all dimensions.

Moreover, open questions were followed to gain participants' thoughts. The results revealed that most participants had experience of running a red light (15 out of 18). Some participants thought the waiting processes were boring and they would use their smartphones to kill time (10 out of 18). When LookMe was placed at the intersections, most participants believed that it could attract their attention, making them relaxed and more patient (14 out of 18). Half of the participants still claimed that they would be less likely to run a red light with LookMe (9 out of 18). Therefore, results from open questions also indicated that LookMe had positive effects on participants' moods and behaviors. The mechanisms that participants were less likely to run a red light might be related to the positive effect of LookMe on their moods and experience while waiting.

\section{Discussions}

This study used user-centered design to explore the possibilities of applying information systems to enhance pedestrians' safety in China. We conducted interviews of pedestrians and experts to better understand pedestrians' current behaviors and direction of possible solutions. Based on the results, we proposed a prototype named LookMe and conducted user tests to investigate user experience. Results from user tests suggest that LookMe can have positive effects on pedestrians' safe behaviors through attracting their attention. In summary, the study found the following points. 
TABLE 3: Questionnaire constructs and items.

\begin{tabular}{|c|c|c|c|c|c|}
\hline Constructs & \multicolumn{5}{|c|}{ Items } \\
\hline Experience & \multicolumn{5}{|c|}{$\begin{array}{l}\text { 1: Please evaluate the degree of boredom that you feel when waiting for the green light with the screen including (not } \\
\text { including) multi-media information. } \\
\text { 2: Please evaluate the degree of monotony that you feel about the environment when waiting for the green light with } \\
\text { screens including (not including) multi-media information. } \\
\text { 3: Please evaluate the degree of impatience that you feel when waiting for the green light with the screen including (not } \\
\text { including) multi-media information. } \\
\text { 4: Please evaluate the degree of willingness that you want to look at the screen including multi-media information when } \\
\text { you are waiting for the green light. }\end{array}$} \\
\hline $\begin{array}{l}\text { Information } \\
\text { need }\end{array}$ & \multicolumn{5}{|c|}{$\begin{array}{l}\text { 1: What type of information you want to see on the multi-media screen? (news, traffic information, weather } \\
\text { information, advertisements, others) } \\
\begin{array}{c}\text { 2: Please evaluate the traffic light information (news, maps, text messages, news, advertisements, videos) in terms of } \\
\text { attractiveness, meaningfulness, and willingness to use. }\end{array}\end{array}$} \\
\hline Open questions & \multicolumn{5}{|c|}{$\begin{array}{l}\text { 1: What do you do when you cross the road? What's your mood? When will you choose to run a red light? } \\
\text { 2: What's the effect of the information system on your behaviors? What's the effect of the information system on your } \\
\text { mood? What's the effect of the information system on your idea of running a red light? }\end{array}$} \\
\hline \multirow{2}{*}{ Items } & Without multimedia & \multicolumn{2}{|c|}{ With multimedia } & \multirow{2}{*}{$t$} & \multirow[b]{2}{*}{$p$} \\
\hline & Mean $\quad$ SD & Mean & SD & & \\
\hline Boredom & 3.61 & 2.11 & 0.58 & 8.10 & $<0.001$ \\
\hline Monotony & 3.67 & 2.11 & 0.76 & 5.10 & $<0.001$ \\
\hline Impatience & 3.06 & 2.00 & 0.69 & 3.70 & $=0.002$ \\
\hline
\end{tabular}

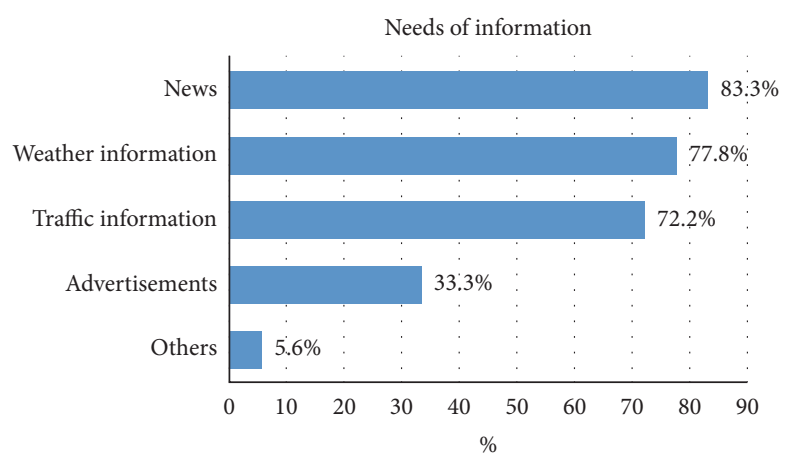

FIGURE 5: Participants' needs of different types of information.

6.1. The Interviews of Pedestrians. Pedestrians' unsafe behaviors in China mainly included running red lights and use of smartphones while walking and crossing. The design of the intersection and sidewalk still needs optimization.

6.2. The Interviews of the Experts. Pedestrians required special attention in transportation. More products and measures were needed to guide pedestrians to conduct safe behaviors.

6.3. Design of LookMe. We proposed an information system called "LookMe" to provide multimedia information to attract pedestrians' attention from using mobile phones and to encourage pedestrians to obey the traffic rules at intersections.
6.4. User Tests. Participants had a relatively high acceptance rate of LookMe. They wanted to obtain diverse information on LookMe including news, weather, and traffic information; but they preferred the screen of LookMe to not include advertisements. LookMe can alleviate their boredom as well as impatience, thereby resulting in lower levels of impulsion to run a red light. Although LookMe is still a prototype, the results have shown its potential to be an effective solution in enhancing pedestrians' safety.

Previous researches on improving pedestrians' safety mainly proposed the systems to detect or alert the pedestrians of the hazards on mobile phones [19-22]. There were limited user tests to investigate the effectiveness of these systems. Different from these systems, this study is more in line with the Safe System approach $[1,23]$. This study conducted interviews to analyze pedestrians' needs, proposed LookMe, and conducted user tests to investigate the effectiveness of LookMe. 


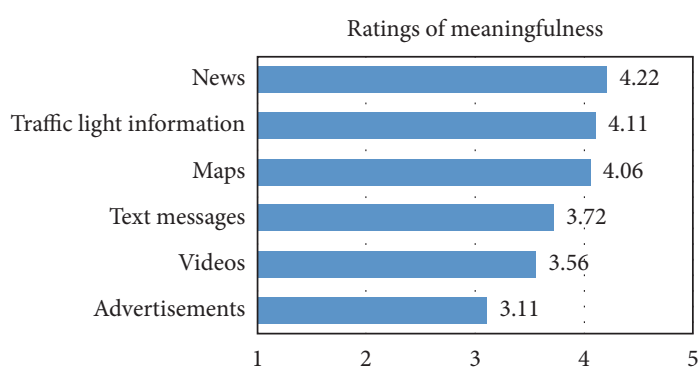

(a)

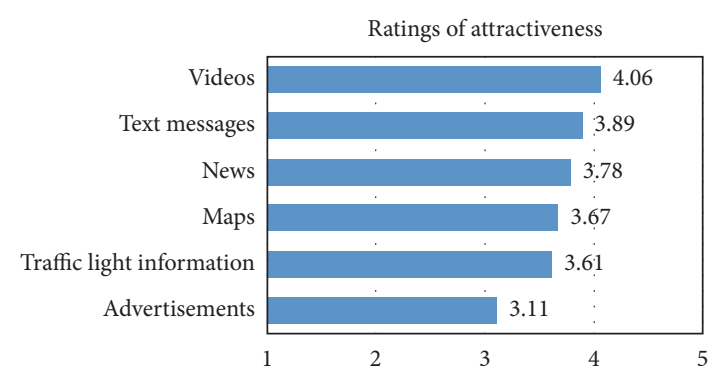

(b)

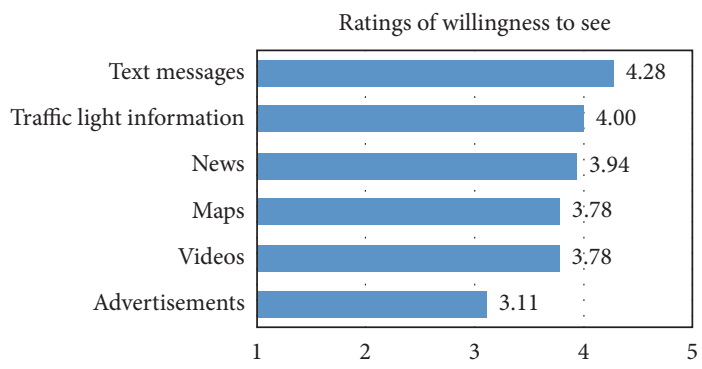

(c)

Figure 6: Participants' ratings of information in terms of meaningfulness (a), attractiveness, (b), and willingness to use (c).

LookMe was a new road infrastructure, which offered more information to pedestrians, collected more data, and guided pedestrians to obey the traffic rules. The results of user tests indicated that participants had high acceptance level of LookMe, and LookMe could alleviate their boredom, impatience, and impulsion to run a red light.

This study has several limitations. First, it used prototypes to investigate the pedestrian's acceptance of LookMe. The prototypes were efficient at the initial stage of the design. Future research could include prototype with higher levels of fidelity or the actual product in user tests to investigate the pedestrian's acceptance of LookMe and to identify the design details. Second, the sample size in user tests was small, though adequate for explorative stage of a new product according to the rule of user tests. Future studies could recruit more participants to improve the design of LookMe based on the rule of power and sample size estimation. Third, our data collection was limited to one Chinese city. The results may hold for other Chinese cities owing to the similar cultural grounds and traffic rules. It would be beneficial to compare results from different countries to identify the cultural effect on pedestrian's behavior and the possible ways to enhance pedestrian's safety. Moreover, LookMe is a new device at the intersections. The feasibility of integrating LookMe with current road infrastructure resources still needs verification.

\section{Data Availability}

The data used to support the findings of this study are available from the corresponding author upon request.

\section{Conflicts of Interest}

The authors declare that they have no conflicts of interest.

\section{References}

[1] World Health Organization, Pedestrian Safety: A Road Safety Manual for Decision-Makers and Practitioners, World Health Organization, Geneva, Switzerland, 2013.

[2] GBD, "The global burden of disease," 2016.

[3] J. Sills, H. Huang, F. Chang et al., "Improve traffic death statistics in China," Science, vol. 362, no. 6415, p. 650, 2018.

[4] A. V. Moudon, L. Lin, J. Jiao, P. Hurvitz, and P. Reeves, “The risk of pedestrian injury and fatality in collisions with motor vehicles, a social ecological study of state routes and city streets in king county, Washington," Accident Analysis \& Prevention, vol. 43, no. 1, pp. 11-24, 2011.

[5] L. Jian, Y. Lizhong, and Z. Daoliang, "Simulation of Bi-direction pedestrian movement in corridor," Physica A: Statistical Mechanics and its Applications, vol. 354, pp. 619-628, 2005.

[6] S. Sudipta, T. Richard, and J. Hunt, "Logistic regression model of risk of fatality in vehicle-pedestrian crashes on national highways in Bangladesh," Transportation Research Record, vol. 2264, no. 1, pp. 128-137, 2011.

[7] T. J. Bungum, C. Day, and L. J. Henry, "The association of distraction and caution displayed by pedestrians at a lighted crosswalk," Journal of Community Health, vol. 30, no. 4, pp. 269-279, 2005.

[8] X. Wang, R. Yu, and C. Zhong, "A field investigation of redlight-running in shanghai, China," Transportation Research Part F: Traffic Psychology and Behaviour, vol. 37, pp. 144-153, 2016.

[9] F. Yan, B. Li, W. Zhang, and G. Hu, "Red-light running rates at five intersections by road user in changsha, China: an observational study," Accident Analysis \& Prevention, vol. 95, pp. 381-386, 2016.

[10] G. Ren, Z. Zhou, W. Wang, Y. Zhang, and W. Wang, "Crossing behaviors of pedestrians at signalized intersections," Transportation Research Record: Journal of the Transportation Research Board, vol. 2264, no. 1, pp. 65-73, 2011. 
[11] M. Brosseau, S. Zangenehpour, N. Saunier, and L. MirandaMoreno, "The impact of waiting time and other factors on dangerous pedestrian crossings and violations at signalized intersections: a case study in montreal," Transportation Research Part F: Traffic Psychology and Behaviour, vol. 21, pp. 159-172, 2013.

[12] E. B. Ebel, C. R. Ayyagari, P. F. Rivara, and L. L. Thompson, "Impact of social and technological distraction on pedestrian crossing behaviour: an observational study," Injury Prevention: Journal of the International Society for Child and Adolescent Injury Prevention, vol. 19, no. 4, pp. 232-237, 2013.

[13] M. Hou, J. Cheng, F. Xiao, and C. Wang, "Distracted behavior of pedestrians while crossing street: a case study in China," International Journal of Environmental Research and Public Health, vol. 18, no. 1, p. 353, 2021.

[14] T. Gandhi and M. M. Trivedi, "Pedestrian protection systems: issues, survey, and challenges," IEEE Transactions on Intelligent Transportation Systems, vol. 8, no. 3, pp. 413-430, 2007.

[15] J. Hatfield and S. Murphy, "The effects of mobile phone use on pedestrian crossing behaviour at signalised and unsignalised intersections," Accident Analysis \& Prevention, vol. 39, no. 1, pp. 197-205, 2007.

[16] H. Zhang, C. Zhang, Y. Wei, and F. Chen, "The effects of mobile phone use on pedestrian crossing behavior and safety at unsignalized intersections," in Proceedings of the 2017 4th International Conference on Transportation Information and Safety (ICTIS), Banff, AB, Canada, August 2017.

[17] M. B. Neider, J. S. McCarley, J. A. Crowell, H. Kaczmarski, and A. F. Kramer, "Pedestrians, vehicles, and cell phones," Accident Analysis \& Prevention, vol. 42, no. 2, pp. 589-594, 2010.

[18] J. Mwakalonge, S. Siuhi, and J. White, "Distracted walking: examining the extent to pedestrian safety problems," Journal of Traffic and Transportation Engineering, vol. 2, no. 5, pp. 327-337, 2015.

[19] Y. Li, F. Xue, X. Fan, Z. Qu, and G. Zhou, "A pedestrian walking safety system based on smartphone built-in sensors," IET Communications, vol. 12, no. 6, pp. 751-758, 2018.

[20] J. Wen, J. Cao, and X. Liu, "We help you watch your steps: unobtrusive alertness system for pedestrian mobile phone users," in Proceedings of the Pervasive Computing and Communications (PerCom), 2015 IEEE International Conference on, pp. 105-113, IEEE, St. Louis, MO, USA, March 2015.

[21] X. D. Yang, K. Hasan, N. Bruce, and P. Irani, “Surround-See: Enabling peripheral vision on smartphones during active use," in Proceedings of the Acm Symposium on User Interface Software \& Technology, pp. 291-300, St. Andrews Scotland, UK, October 2013.

[22] S. Hu, Su Lu, H. Liu, H. Wang, F. Tarek, and Abdelzaher, "Smartroad: smartphone-based crowd sensing for traffic regulator detection and identification," ACM Transactions on Sensor Networks, vol. 11, no. 4, p. 55, 2015.

[23] European Conference of Ministers of Transport, Towards Zero. Ambitious Road Safety Targets and the Safe System Approach, European Conference of Ministers of Transport, Brussels, Belgium 2008.

[24] K. Vasileiou, J. Barnett, S. Thorpe, and T. Young, "Characterising and justifying sample size sufficiency in interviewbased studies: systematic analysis of qualitative health research over a 15-year period," BMC Medical Research Methodology, vol. 18, no. 1, p. 148, 2018.

[25] A. Bogner, B. Littig, and W. Menz, Introduction: Expert Interviews - an Introduction to a New Methodological Debate, Springer, Berlin, Germany, 2009.
[26] W. Hwang and G. Salvendy, "Number of people required for usability evaluation," Communications of the ACM, vol. 53, no. 5, pp. 130-133, 2010.

[27] L. Ha, "Advertising clutter in consumer magazines: dimensions and effects," Journal of Advertising Research, vol. 36, no. 4, pp. 76-85, 1996.

[28] A. E. Hühn, V.-J. Khan, P. Ketelaar et al., "Does location congruence matter? A field study on the effects of locationbased advertising on perceived ad intrusiveness, relevance \& value," Computers in Human Behavior, vol. 73, 2017.

[29] S. Mccoy, A. Everard, D. F. Galletta, and G. D. Moody, "Here we go again! The impact of website ad repetition on recall, intrusiveness, attitudes, and site revisit intentions," Information \& Management, vol. 54, no. 1, pp. 14-24, 2016. 